\title{
Nucleus capture by macroscopic dark matter
}

\author{
Yang $\mathrm{Bai}^{a}$ and Joshua Berger ${ }^{b}$ \\ ${ }^{a}$ Department of Physics, University of Wisconsin-Madison, \\ University Avenue, Madison, Wisconsin, U.S.A. \\ ${ }^{b}$ Department of Physics and Astronomy, University of Pittsburgh, \\ O'Hara Street, Pittsburgh, Pennsylvania, U.S.A. \\ E-mail: yangbai@physics.wisc.edu, josh.berger@pitt.edu
}

ABSTRACT: For a class of macroscopic dark matter with a large interaction strength with Standard Model particles, a nucleus could be captured by the dense, heavy dark matter as it traverses ordinary material. The radiated photon carries most of the binding energy and is a characteristic signature for dark matter detection. We develop analytic formulas and present numerical results for this radiative capture process in the low energy, non-dipole limit. Large-volume neutrino detectors like NO $\nu$ A, JUNO, DUNE and Super(Hyper)-K may detect multi-hit or single-hit radiative capture events and can search for dark matter up to one gram in mass.

Keywords: Beyond Standard Model, Neutrino Physics, Solitons Monopoles and Instantons

ARXIV EPRINT: 1912.02813 


\section{Contents}

1 Introduction 1

2 Example macroscopic dark matter: EWS-DMB 3

3 Radiative capture cross section 4

3.1 Bound states $\quad 5$

$\begin{array}{lll}3.2 & \text { Scattering states } & 7\end{array}$

3.3 General scattering amplitude $\quad 8$

3.4 Dipole limit 9

$\begin{array}{ll}3.5 & \text { Low energy limit }\end{array}$

$\begin{array}{llr}3.6 & \text { Large radius limit } & 12\end{array}$

4 Prospects for detection $\quad 14$

5 Discussion and conclusions $\quad 17$

\section{Introduction}

Macroscopic dark matter (MDM) is a general class of models with dark matter (DM) in a compact and composite state with a large radius and mass. The composite consists of many elementary dark matter particles and has dramatically different properties from a microscopic particle. In the literature, MDM appears in many scenarios either within the Standard Model (SM) or beyond. For instance, the massive astrophysical compact halo object (MACHO) such as brown dwarfs is made of SM particles and has long been proposed as a dark matter candidate [1,2], although their abundance is constrained by microlensing experiments to occupy only a small fraction of dark matter [3]. For lighter MDM below the microlensing threshold mass around $10^{-11} M_{\odot}$ [4], one could have socalled quark nuggets that are made of quark matter in the unconfined QCD phase [5-7]. These objects have a QCD scale energy density, $\sim 10^{15} \mathrm{~g} / \mathrm{cm}^{3}$, and a radius $\sim 0.01 \mathrm{~cm}$. Beyond the SM, MDM can be composed of bosonic constituents, as in non-topological solitons [8-10]. For example, in ref. [11], electroweak symmetric dark matter balls (EWSDMB) were proposed as a MDM candidate in the simple Higgs-portal dark matter model with an electroweak scale energy density, $\sim 10^{27} \mathrm{~g} / \mathrm{cm}^{3}$ and a radius $\sim 10^{-8} \mathrm{~cm}$. Other than quark nuggets or EWS-DMBs with well-defined QCD or electroweak interactions with SM particles, there are also other MDM models with only gravitational interactions or other unknown interactions with ordinary matter, including dark quark nuggets [12], asymmetric dark matter nuggets [13] and dark blobs [14] (for a recent review see [15]).

Some MDM candidates like QCD quark nuggets and EWS-DMBs have relatively large interaction cross sections with ordinary matter. QCD quark nuggets behave basically like a very heavy nucleus. When they elastically scatter off a nucleus on the target, various nearby 
bound states of MDM and nucleus enhance the scattering process, leading to a geometric size cross section at large radius. Similarly, for EWS-DMBs, the nucleus has slightly different masses inside and outside the dark matter state. For a large radius $R_{(1)}$ above the bound state threshold radius, the elastic scattering cross section saturates the geometric one and varies between $2 \pi R_{\text {(7) }}^{2}$ and $4 \pi R_{(\text {(क) }}^{2}$ up to quantum mechanical shadowing effects [11]. This elastic scattering cross section is rather large such that the MDM may interact with nuclei multiple times in a detector. Traditional dark matter direct detection experiments that look for a single-hit event may veto the MDM-induced multi-hit events and may not be suitable to search for "strongly interacting" $\operatorname{MDM}[16,17]$. Because of the spectacular multi-hit signature, potential experiments searching for MDM do not necessarily need to be located underground. The key requirement is to have a large volume detector to compensate the smallness of the MDM flux. Indeed, the large neutrino detectors like Borexino, ICARUS, NO $\nu$ A, JUNO, Super-Kamiokande (Super-K), DUNE, Hyper-Kamiokande (Hyper-K) or even IceCube could be used to search for MDM. On the hand, the trigger thresholds of the neutrino detectors become crucial because the individual MDM scatterings do not deposit that much energy. For instance, Borexino and JUNO could have a sufficiently low threshold energy to detect some multi-hit scattering events [11], but not for the other larger volume experiments, which have energy thresholds of at least $1 \mathrm{MeV}$.

In this paper, rather than studying elastic scattering events of MDM, we point out another interesting MDM-induced signature. As MDM hits a nucleus in the detector, the nucleus and MDM could form one of many bound states, as mentioned in ref. [11] for EWS-DMBs. Just like hydrogen formation from an electron and a proton, $e^{-}+p \rightarrow \mathrm{H}+\gamma$, the radiative capture or recombination process can generate a photon in the final state. The photon carries most of the binding energy and could have an energy higher than the kinetic energy of the scattering system. Similarly, the nucleus radiative capture process by a MDM, ${ }_{\mathrm{Z}}^{\mathrm{A}} \mathrm{N}+\mathrm{MDM} \rightarrow$ bound states $+\gamma$, can produce energetic photons in the final state. Depending on the detailed properties of the MDM, the photons produced could have an energy above $1 \mathrm{MeV}$ or even up to $\mathrm{GeV}$, which could be observed by a larger volume neutrino detector.

The calculation for the radiative capture cross section can be found in several systems. Other than the hydrogen recombination process $[18,19]$ with a long-range Coulomb force, the capture of a nucleon or nucleus by a grand unified theory magnetic monopole has been studied in [20]. For both cases, because of the radiated photon wavelength is much longer than the Bohr radius, the dipole approximation has been used to simplify the calculation. The situation is different for a MDM with a hard-sphere structure. The released photon energy $q$ could be so energetic such that its wavelength $2 \pi / q$ could be much shorter than the MDM size $R_{\oplus}$. Therefore, a non-dipole calculation is needed to study the radiative capture process of MDM with a large $R_{(\rightarrow)}$. The situation is similar to the neutron capture by a nucleus, although the underlying interactions are different and one usually relies on numerical tools to estimate the cross sections [21]. In this paper, without relying on dipole approximation, we take the low energy limit with a small scattering momentum $k$ such that $k R_{\oplus} \ll 2 \pi$, perform an analytic calculation, and extrapolate the radiative capture cross section to a large radius beyond this approximation. 
Our paper is organized as follows. We first use the EWS-DMB as a concrete MDM example to set up the stage for our calculation in section 2. Section 3 contains the main calculations with the dipole limit in subsection 3.4, low energy limit in subsection 3.5 and extrapolation to a large radius in subsection 3.6. The prospects of detection in various neutrino detectors are discussed in section 4. We summarize our results in section 5 .

\section{Example macroscopic dark matter: EWS-DMB}

We use EWS-DMB as a working example to discuss the radiative capture of nuclei by macroscopic dark matter. Our analysis can be also applied to other types of MDM that have a large interaction strength with SM particles. As discussed in ref. [11], there exists a non-topological soliton state for dark matter in the simple Higgs-portal dark matter model with an unbroken $\mathrm{U}(1)_{\Phi}$ dark matter number symmetry. The relevant interactions beyond the SM have

$$
\mathcal{L} \supset-\lambda_{\phi h} \Phi^{\dagger} \Phi H^{\dagger} H-m_{\phi, 0}^{2} \Phi^{\dagger} \Phi-\lambda_{\phi}\left(\Phi^{\dagger} \Phi\right)^{2}
$$

with $\lambda_{\phi h}$ as the Higgs-portal interaction, $m_{\phi, 0}$ as the dark matter bare mass independent of electroweak (EW) symmetry breaking, $\lambda_{\phi}$ as the self-interaction of dark matter particle $\Phi$. After EW symmetry breaking, the free dark matter particle mass is $m_{\phi}=\left(m_{\phi, 0}^{2}+\right.$ $\left.\lambda_{\phi h} v_{\mathrm{EW}}^{2} / 2\right)^{1 / 2}$ with $v_{\mathrm{EW}}=246 \mathrm{GeV}$, the $\mathrm{EW}$ vacuum expectation value (VEV).

In addition to the free particle dark matter state, there is a non-topological soliton state of dark matter with a charge $Q=i \int d x^{3}\left(\Phi^{\dagger} \partial_{t} \Phi-\Phi \partial_{t} \Phi^{\dagger}\right)$. Solving the classical equations of motion for both $\Phi$ and $H$ and in the large $Q$ limit, the dark matter soliton state has a mass of

$$
M_{\oplus}=Q \omega_{c}=Q\left[m_{\phi, 0}^{2}+\left(\lambda_{\phi} / 4 \lambda_{h}\right)^{1 / 2} m_{h}^{2}\right]^{1 / 2}
$$

Here, $\lambda_{h} \approx 0.13$ is the Higgs quartic coupling in the SM and $m_{h} \approx 125 \mathrm{GeV}$ is the Higgs boson mass. In the parameter region with $\sqrt{\lambda_{\phi}} / \lambda_{\phi h}<1.4$, one has $\omega_{c}<m_{\phi}$, so the soliton state has a lighter mass per charge than a free dark matter particle state. For a non-negligible $\lambda_{\phi}$ and a spherically symmetric EWS-DMB, the self-interaction of $\Phi$ field induces a step-like or hard-sphere profile for the $\Phi$ field up to a radius $R_{\oplus}$ and a wall thickness of $1 / v_{\mathrm{EW}}$. In the large $Q$ limit, there are simple scaling laws between the DMBs charge, size and mass: $M_{\text {(क) }} \sim Q \sim R_{\text {(D) }}^{3}$. The energy density of a DMB is

$$
\rho_{\text {(Ф) }}=\frac{M_{\text {(7) }}}{(4 \pi / 3) R_{\text {(7) }}^{3}} \sim v_{\mathrm{EW}}^{4} \sim(100 \mathrm{GeV})^{4}
$$

which is much denser than ordinary matter. The early universe production of the EWSDMB from a first-order phase transition has also been discussed in ref. [11]. The EWSDMBs can have a macroscopic mass above 1 gram and a radius above $10^{5} \mathrm{GeV}^{-1}$, dramatically above the electroweak scale. The masses and radii of EWS-DMBs are very sensitive to the portal coupling strength $\lambda_{\phi h}$, which is also responsible for providing the first-order electroweak phase transition. In the range of $\lambda_{\phi h}$ from 2 to 9 , the average DMB mass 
ranges from $1 \times 10^{24} \mathrm{GeV}$ and $9 \times 10^{33} \mathrm{GeV}$ [11]. There is no collider constraint on the model parameter space with $\lambda_{\phi h}>2$. For a large coupling, the $\Phi$ particle has a mass above half the Higgs mass and has a suppressed pair-production cross section from an off-shell Higgs boson at the Large Hadron Collider.

Due to the interplay of $\Phi$ and Higgs profiles, the field value of $\Phi$ in the inner region of EWS-DMB is large enough to flip the sign of the effective Higgs mass squared, $\lambda_{\phi h} \Phi^{\dagger} \Phi-\lambda_{h} v_{\mathrm{EW}}^{2}$, and prefers a zero Higgs VEV or unbroken electroweak symmetry. Hence, this soliton state is an interesting macroscopic dark matter, because it sustains an EW symmetric "vacuum" in a finite region of space, immersed in the normal EW breaking vacuum.

When DMB with a large radius scatters with a nucleon or a nucleus, a large scattering cross section is generically anticipated. For elastic scattering, there are effects due to shallow bound states at several partial waves. After summing over these partial waves, the cross section follows a "hard ball" behavior, between 2 and 4 times the geometric cross section [11]. Multi-hit signals are the characteristic features of the DMB elastic scattering events. Since only $\mathcal{O}(10 \mathrm{keV})$ are anticipated from each scattering, a low energy threshold below around $1 \mathrm{MeV}$ is required to identify the dark matter scattering events. In this paper, we will instead concentrate on the important radiative capture process, which can convert the binding energy of a nucleus and a DMB into photons with energies of $\mathcal{O}(1 \mathrm{MeV}-100 \mathrm{MeV})$, depending on nucleus mass number.

\section{Radiative capture cross section}

A nucleus can be captured by a DMB while emitting a photon in a process similar to neutron radiative capture by a nucleus, such as $n+{ }_{79}^{197} \mathrm{Au} \rightarrow{ }_{79}^{198} \mathrm{Au}+\gamma$. Explicitly, the DMB-induced radiative capture process is

$$
{ }_{\mathrm{Z}}^{\mathrm{A}} \mathrm{N}+\Phi \rightarrow \Phi+\gamma
$$

with $\Phi \mathrm{N}$ as a bound state of DMB and a nucleus. For the neutron capture case, depending on the incident neutron kinetic energy, there is a low energy region with $1 / v$ scaling, an intermediate resonant region and a fast neutron region. For the DMB case, the relative speed between the DMB and the target nucleus in a laboratory is roughly the averaged dark matter speed in our local galaxy or around $v \sim 300 \mathrm{~km} / \mathrm{s} \approx 10^{-3} c$. Using the reduced mass $\mu \approx A m_{p}$ and the proton mass $m_{p}=0.938 \mathrm{GeV}$, the kinetic energy of the scattering process is $E_{\text {kin }} \approx k^{2} /(2 \mu) \approx m_{A} v^{2} / 2 \approx A \times 0.5 \mathrm{keV}$, which does not vary too much for comparable nucleus mass number. On the other hand, we do not know the exact radius of DMB and will keep the radius as a free parameter of the model. As with neutron radiative capture, we will see three qualitatively different regions: no bound state formation, resonant scattering and geometric cross section saturation.

Though the radiated photon of course requires a relativistic description, both the initial scattering state and final bound state are well described by non-relativistic mechanics. For a heavy DMB with $M_{\oplus} \gg m_{A}$, the center-of-mass frame is approximately the rest frame of DMB. Effectively, one can think that a nucleus enters the inner region of DMB. 
For simplicity, we only consider spherically symmetric DMBs in this work. The change in the profile of the Higgs field from non-zero outside to zero inside is rapid, forming a "wall" of thickness roughly $\pi / v_{\mathrm{EW}}^{-1} \ll R_{\text {(D) }}$ [11]. This change in the Higgs VEV modifies the contribution of the quark masses to the mass of the nucleus, which is to excellent approximation the reduced mass of the system. The shift of the nucleus mass from outside to inside of the DMBs is given by

$$
\Delta m_{A} \approx-A y_{h N N} v_{\mathrm{EW}}
$$

where $A$ is the mass number of the nucleus and $y_{h N N}$ is the nucleon Yukawa coupling, which is roughly the same for protons and neutrons and has a value of $1.1 \times 10^{-3}$ [22, 23]. The shift in the mass is small since the contribution of the Higgs-generated quark masses to the nucleon masses is small compared to that of QCD dynamics. A non-relativistic expansion of the Klein-Gordon equation for the nucleus in the background of the changing Higgs VEV profile through the spherically symmetric DMB can be performed. Note that physical fields of any Lorentz representation nevertheless satisfy the Klein-Gordon equation. The spin degrees of freedom decouple in the non-relativistic limit. Thus, the Schrödinger analysis applies to nuclei of any spin.

If the expansion is done around the outside mass $Z m_{p}+(A-Z) m_{n}$, the changing Higgs VEV generates a spherical potential well for the nucleus inside the DMB in the "thin wall" approximation. The potential has a depth of

$$
V_{0}=-\Delta m_{A} .
$$

We allow for some uncertainty in the determination of this effective potential due to nuclear effects as well as other models different from the baseline EWS-DMB model and with a suppressed modification on the nucleus mass. More concretely and in our later numeric calculation, we will use $V_{0}=A \times 32 \mathrm{MeV}$, which is around one eighth of the maximal electroweak restored case. The scattering cross section becomes a "textbook" one, although we are not aware of the relevant results in the large radius parameter region, where the usual dipole approximation breaks down. In the DMB-nucleus center-of-mass frame, the relevant Schrödinger equation is

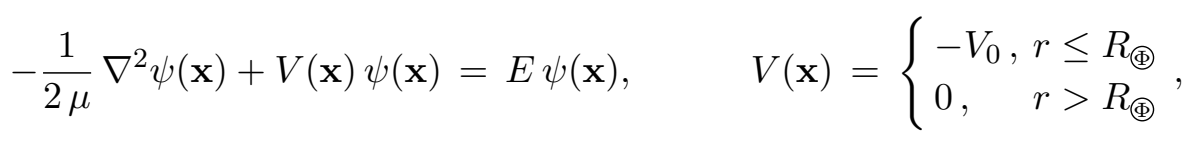

where $r=|\mathbf{x}|$ is the magnitude of the center of mass coordinate.

\subsection{Bound states}

For bound states, i.e. states with $E<0$, we write the wave functions in the spherical coordinate with quantum numbers $n, \ell, m$

$$
\psi_{n \ell m}(\mathbf{x})=R_{n \ell}(r) Y_{\ell m}(\hat{\mathbf{x}}) .
$$

Here, $Y_{\ell m}(\hat{\mathbf{x}})$ is a spherical harmonic. The bound state is normalized as $\int d^{3} x\left|\psi_{n \ell m}(\mathbf{x})\right|^{2}=$ 1. The normalizable radial wave functions can be expressed in terms of spherical Bessel 

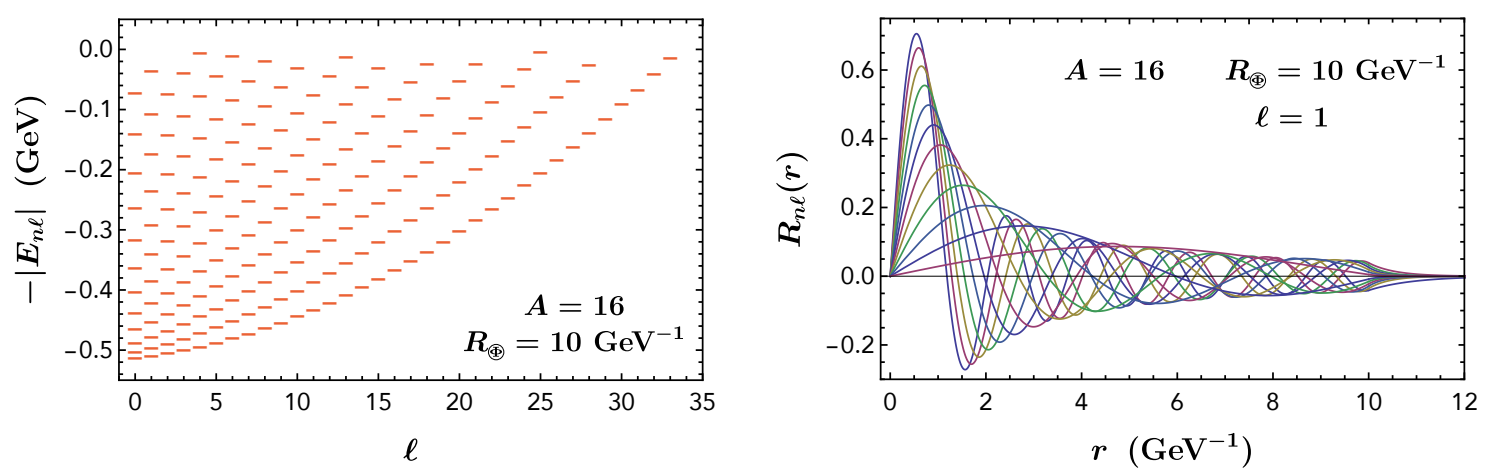

Figure 1. Left panel: the energy levels for nucleus-DMB bound states for different partial wave numbers $\ell$. Right panel: the 12 radial wave functions as a function for $r$ for the $p$-wave bound states with $\ell=1$.

functions

$$
R_{n \ell}(r)= \begin{cases}R_{n \ell}^{\mathrm{in}}(r)=d_{1} j_{\ell}\left(\kappa_{n \ell} r\right), & r \leq R_{\text {(क) }}, \\ R_{n \ell}^{\text {out }}(r)=d_{2}\left[j_{\ell}\left(i k_{n \ell} r\right)+i y_{\ell}\left(i k_{n \ell} r\right)\right], & r>R_{\text {(क) }},\end{cases}
$$

with $\kappa_{n \ell}=\sqrt{2 \mu\left(V_{0}-\left|E_{n \ell}\right|\right)}$ and $k_{n \ell}=\sqrt{2 \mu\left|E_{n \ell}\right|}$. The coefficients $d_{1,2}$ and the energy eigenvalues $E_{n \ell}$ are determined by the boundary conditions $R_{n \ell}^{\text {in }}\left(R_{\oplus}\right)=R_{n \ell}^{\text {out }}\left(R_{\oplus}\right)$, $R_{n \ell}^{\text {in }}\left(R_{(\Phi)}\right)=R_{n \ell}^{\text {out }}\left(R_{(\Phi)}\right)$ and the normalization condition $\int_{0}^{\infty} d r r^{2} R_{n \ell}^{2}(r)=1$. While the energy eigenvalue equation cannot be solved analytically without any approximations, the coefficients $d_{1}$ and $d_{2}$ are found to be

$$
d_{1}=\frac{1}{N_{n \ell} j_{\ell}\left(\kappa_{n \ell} R_{(\Phi)}\right)}, \quad d_{2}=\frac{1}{N_{n \ell}\left[j_{\ell}\left(i k_{n \ell} R_{(\Phi)}\right)+i y_{\ell}\left(i k_{n \ell} R_{(\Phi)}\right)\right]},
$$

where

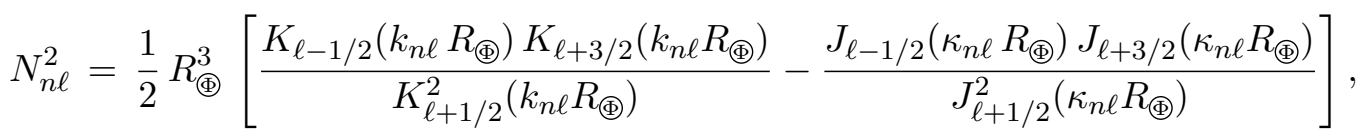

in terms of Bessel functions $J_{\nu}$ and $K_{\nu}$.

For each partial wave $\ell$, there is a threshold radius $R_{\mathrm{th}}^{\ell}$ below which there are no bound states. The threshold is given by

$$
R_{\mathrm{th}}^{\ell}=\frac{\pi}{2 \sqrt{2 \mu V_{0}}} J_{\ell-1 / 2,1}
$$

with $J_{\nu, 1}$ as the first zero of the Bessel function $J_{\nu}$. For example, one has $R_{\mathrm{th}}^{0}=0.41 \mathrm{GeV}^{-1}$ and $R_{\mathrm{th}}^{1}=0.80 \mathrm{GeV}^{-1}$ for $A=16$. For a large radius $R_{\text {(क) }}$, many bound states exist. For $A=16$ and $R_{\oplus}=10 \mathrm{GeV}^{-1}$, we show the energy levels in the left panel and the radial wave functions for $\ell=1$ in the right panel of figure 1 . There are totally 194 bound states, including $12 s$-wave and $12 p$-wave bound states. For more excited bound states with a smaller value of $\left|E_{n \ell}\right|$, there are more nodes in the wave function. 
In the limit that $k_{n \ell} R_{(\Phi)} \gg 1$, the bound state wave function outside the ball is exponentially small. In this limit, the bound state solution is well-approximated by the infinite well solution, for which $R_{n \ell}^{\text {out }} \approx 0$. The energy eigenvalues are given by the condition $\kappa_{n \ell}=J_{\ell+1 / 2, n} / R_{\text {(क) }}$, where $J_{\ell+1 / 2, n}$ are the Bessel function zeroes. For $n \gg \ell$, these wavenumbers are well approximated by $\kappa_{n \ell} \approx[\pi(n+\ell / 2-1 / 4)] / R_{\text {(7) }}$.

\section{2 $\quad$ Scattering states}

The scattering state is also an eigenstate of energy, though with $E=k^{2} /(2 \mu)>0$. It is not an eigenstate of angular momentum as the scattering state is incident from far away with fixed momentum. The incident scattering wave function has the form $e^{i \mathbf{k} \cdot \mathbf{x}}$ far from the potential. As angular momentum is conserved in scattering off of a spherical potential, the full wave function can be decomposed as

$$
\psi_{\mathbf{k}}(\mathbf{x})=\sum_{\ell m} R_{k \ell}(r) Y_{\ell m}^{*}(\hat{\mathbf{k}}) Y_{\ell m}(\hat{\mathbf{x}})
$$

The radial wave function can also be expressed in terms of spherical Bessel functions,

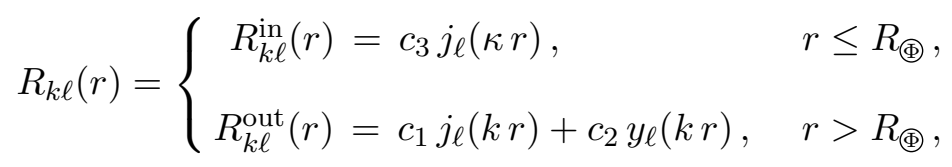

where $\kappa=\sqrt{k^{2}+2 \mu V_{0}}$ and $k=\sqrt{2 \mu E}$. Unitarity implies that scattering off of a spherical potential leads to a phase shift in the exterior wave function partial waves far away from the ball. In the decomposition of the wave function into partial waves, the phase shift condition corresponds to $\sqrt{c_{1}^{2}+c_{2}^{2}}=4 \pi i^{\ell}$. The remaining combinations of coefficients are determined by the boundary conditions $R_{k \ell}^{\text {in }}\left(R_{(\Phi)}\right)=R_{k \ell}^{\text {out }}\left(R_{(\Phi)}\right)$ and $R_{k \ell}^{\text {in }}\left(R_{\text {(Ф) }}\right)=R_{k \ell}^{\prime \text { out }}\left(R_{\text {(क) }}\right)$. The coefficients for the exterior wave function can be written as

$$
c_{1}=4 \pi i^{\ell} \cos \delta, \quad c_{2}=4 \pi i^{\ell} \sin \delta,
$$

where $\delta$ is the partial wave scattering phase, given by

$$
\tan \delta=\frac{k j_{\ell}\left(\kappa R_{\oplus}\right) j_{\ell+1}\left(k R_{\oplus}\right)-\kappa j_{\ell+1}\left(\kappa R_{\oplus}\right) j_{\ell}\left(k R_{\oplus}\right)}{\kappa j_{\ell+1}\left(\kappa R_{\text {(Ф) }}\right) y_{\ell}\left(k R_{\oplus}\right)-k j_{\ell}\left(\kappa R_{\oplus}\right) y_{\ell+1}\left(k R_{\oplus}\right)} .
$$

For $s$-wave scattering state, the normalization factors have a simple analytic formula. For instance,

$$
c_{3}=\frac{4 \sqrt{2} \pi \kappa}{\sqrt{\kappa^{2}+k^{2}+\left(\kappa^{2}-k^{2}\right) \cos \left(2 \kappa R_{(\Phi)}\right)}} \approx \frac{4 \pi}{\left|\cos \left(\kappa R_{(\Phi)}\right)\right|},
$$

where we have taken the limit of $k \ll \kappa$ for the small kinetic energy $E \ll V_{0}$. So, the amplitude of the inner-region wave function has a large peak value at $R_{(\Phi)}=(2 m+1) \pi /(2 \kappa)$ for integer $m$, which is coincident with the values of $R_{\oplus}$ to have an $s$-wave resonant elastic scattering. In our later calculation of the radiative capture cross section, $\sigma_{\gamma}$, the oscillating peak structure of $c_{3}$ will induce a similar behavior for $\sigma_{\gamma}$ as a function of $R_{\text {(D) }}$. For other partial-wave scattering states, the similar resonant enhancement occurs for certain values of $R_{\text {(T) }} \approx J_{\ell-1 / 2, n} / \kappa$ where $J_{\nu, n}$ are Bessel function zeros. 


\subsection{General scattering amplitude}

We begin by writing the general formula for the scattering amplitude. We then derive the analytic formulas to calculate the cross sections in two interesting limits: the dipole and low energy limits.

The electromagnetic coupling of the nucleus to the vector potential is given by the interaction Hamiltonian

$$
H_{\mathrm{int}}=\frac{1}{2 \mu} Z e\left[\mathbf{p}_{\mathrm{N}} \cdot \mathbf{A}\left(\mathbf{x}_{\mathrm{N}}\right)+\mathbf{A}\left(\mathbf{x}_{\mathrm{N}}\right) \cdot \mathbf{p}_{\mathrm{N}}\right],
$$

where $\mathbf{x}_{\mathrm{N}}$ and $\mathbf{p}_{\mathrm{N}}$ are the nucleus position and momentum operators respectively. In the $M_{\oplus} \gg m_{A}$ limit, these nucleus operators reduce to $\mathbf{X}+\mathbf{x}$ and $\mathbf{p}$, where uppercase letters denote center-of-mass motion and lowercase letters denote relative motion. The scattering matrix element is then given by

$$
\mathcal{M}_{n \ell m}=\frac{1}{2 \mu} Z e \boldsymbol{\epsilon}^{*} \cdot \int d^{3} x e^{-i \mathbf{q} \cdot \mathbf{x}}\left[\nabla \psi_{n \ell m}^{*}(\mathbf{x}) \psi_{\mathbf{k}}(\mathbf{x})-\psi_{n \ell m}^{*}(\mathbf{x}) \nabla \psi_{\mathbf{k}}(\mathbf{x})\right],
$$

where $\boldsymbol{\epsilon}=\boldsymbol{\epsilon}(\mathbf{q})$ is the photon polarization satisfying $\mathbf{q} \cdot \boldsymbol{\epsilon}(\mathbf{q})=0$ and $\psi_{\mathbf{k}} / \psi_{n \ell m}$ are the scattering/bound state wave functions relative to the center of mass respectively. Note that the scattering and bound state wave functions have different normalizations and different mass dimensions. The scattering wave function is not normalizable as the incident wave is a plane wave. The photon momentum and energy have $|\mathbf{q}|=\omega_{n \ell} \approx E_{\mathbf{k}}+\left|E_{n \ell}\right|$. For a small dark matter velocity, the kinetic energy is in general smaller than the binding energy and the photon energy is approximately the binding energy.

The radiative capture cross section in the non-relativistic normalization is then given by

$$
\sigma_{\gamma, n \ell}=\frac{1}{v} \int d \Omega \frac{\left|E_{n \ell}\right|}{8 \pi^{2}} \sum_{m}\left|\mathcal{M}_{n \ell m}\right|^{2} .
$$

In general, one can keep all partial wave functions in the scattering state, expanding $e^{-i \mathbf{q} \cdot \mathbf{x}}$ in partial waves as well and performing the integration to calculate $\sigma_{\gamma, n \ell}$. This procedure is conceptually clear, but practically tedious. Instead, we mainly focus on few parameter regions with good approximation schemes, derive analytic formulas and present the cross sections based on them.

The most relevant parameters for radiative capture of the EWS-DMB is the radius $R_{\text {(D) }}$, the scattering kinetic energy or momentum $k \equiv|\mathbf{k}|$ and the radiated photon energy $q \equiv|\mathbf{q}|$. The three limits are

- Dipole limit: $q R_{(\Phi)} \ll 1$. In this limit, the wavelength of the emitted photon is much larger than the radius of the DMB, so that the wave function of the emitted photon becomes trivial or $e^{-i \mathbf{q} \cdot \mathbf{x}} \rightarrow 1$.

- Low energy limit: $k R_{(\Phi)} \ll 1$. In this limit, only the $s$-wave mode of the scattering state has a significant contribution.

- Semi-classical limit: $q R_{\oplus} \gg 1$ and $k R_{\oplus} \gg 1$. In this limit, a large number of closely spaced bound states can be produced and all wave functions are oscillating rapidly across the potential. 
For the semi-classical limit, we cannot take the classical limit as the dominant radiation effect comes from the suppressed quantum effects over the entire radius of the potential. This limit is the most challenging to compute due to the large number of contributing states and amplitudes. We approximate the cross section via scaling relations inferred from the other two limits as well as the behavior of the neutron capture cross sections by a nucleus in the similar limit.

We also note that the hydrogen radiative capture process satisfies both dipole limit and low energy limit because of the smallness of the electromagnetic coupling and the long-range property of Coulomb interactions. A simple analytic result can therefore be obtained in that case $[18,19,24]$.

\subsection{Dipole limit}

In the dipole limit with $q R_{(\Phi)} \ll 1,|\mathbf{q} \cdot \mathbf{x}| \ll 1$ for all $|\mathbf{x}| \leq R_{(1)}$. In this limit, we simplify the calculation using the operator relation $\mathbf{p}=-i \mu[\mathbf{x}, H]$ [25], where $H$ is the Hamiltonian excluding the interaction (3.15). The matrix element becomes

$$
\left|\mathcal{M}_{n \ell m}\right|=Z e\left(\left|E_{n \ell}\right|+E_{\mathbf{k}}\right) \int d^{3} x \psi_{n \ell m}^{*}(\mathbf{x}) \boldsymbol{\epsilon}^{*} \cdot \mathbf{x} \psi_{\mathbf{k}}(\mathbf{x})
$$

Starting with eq. (3.18), we perform the angular integration over spherical harmonics, square the amplitude, integrate over the photon emission angle, and sum over photon polarizations and $m$ of the bound states to obtain the radiative capture cross section for the bound state $(n, \ell)$

$$
\begin{aligned}
\sigma_{\gamma, n \ell}=\frac{1}{v} & \frac{Z^{2} \alpha}{3 \pi}\left|E_{n \ell}\right|\left(\left|E_{n \ell}\right|+E_{\mathbf{k}}\right)^{2} \\
& \times\left[\ell\left|\int d r r^{3} R_{n \ell}(r) R_{k \ell-1}(r)\right|^{2}+(\ell+1)\left|\int d r r^{3} R_{n \ell}(r) R_{k \ell+1}(r)\right|^{2}\right] .
\end{aligned}
$$

Using the oxygen nucleus as an example, we show the radiative capture cross section as a function of $R_{\text {(क) }}$ in figure 2. Since photon energy can be as large as the depth of potential barrier, $V_{0} \approx A \times 32 \mathrm{MeV}$, and if all deep bound states are included, the dipole approximation is only valid up to a radius of $R_{(\Phi)} \approx 2 \pi / V_{0} \approx 12 \mathrm{GeV}$, which sets the upper end of the $x$-axis in figure 2. The oscillating peak structure is also obvious for this plot. As we discussed around (3.14), the scattering states could be close to a bound state for a certain $R_{\text {(क) }}$, leading to resonant enhancement of the cross section.

\subsection{Low energy limit}

In the low energy limit with $k R_{(\Phi)} \ll 1$, we find it convenient to use integration by parts and the on-shell photon conditions $\mathbf{q} \cdot \boldsymbol{\epsilon}=0$ to rewrite the amplitude as

$$
\mathcal{M}_{n \ell m}=-\frac{1}{\mu} Z e \boldsymbol{\epsilon}^{*} \cdot \int d^{3} x e^{-i \mathbf{q} \cdot \mathbf{x}} \psi_{n \ell m}^{*}(\mathbf{x}) \nabla \psi_{\mathbf{k}}(\mathbf{x}) .
$$




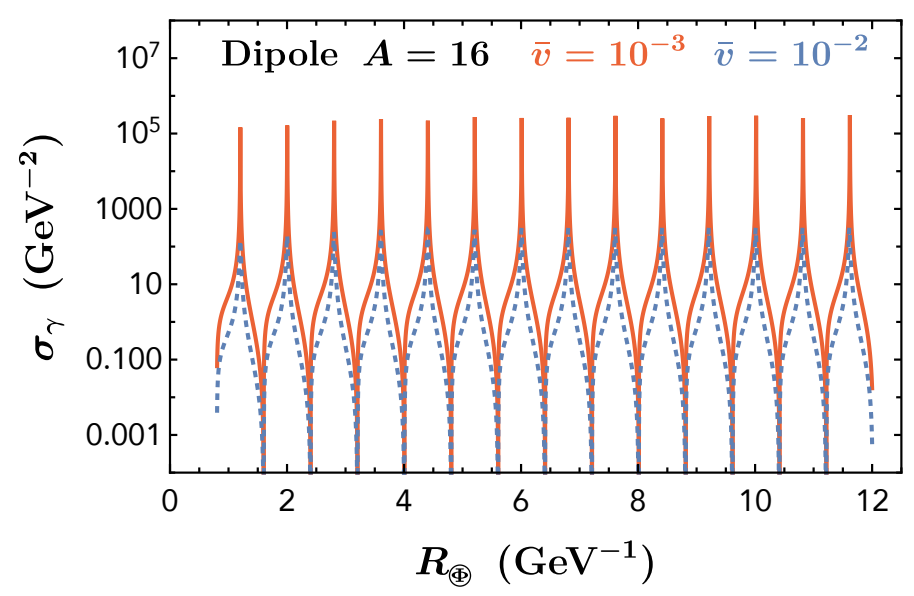

Figure 2. Radiative capture cross section as a function of the DMB radius for the oxygen nucleus with $Z=8$ and $A=16$ in the dipole approximation. The dominant $p$-wave bound states and $s$-wave scattering states are included in this plot. Two different dark matter averaged velocities of $\bar{v}=10^{-3}$ and $\bar{v}=10^{-2}$ are considered.

For the dipole factor $e^{-i \mathbf{q} \cdot \mathbf{x}}$, we decompose its complex conjugate in spherical harmonics as

$$
e^{i \mathbf{q} \cdot \mathbf{x}}=\sum_{\ell^{\prime}, m^{\prime}} 4 \pi i^{\ell^{\prime}} j_{\ell^{\prime}}(q r) Y_{\ell^{\prime} m^{\prime}}^{*}(\hat{\mathbf{q}}) Y_{\ell^{\prime} m^{\prime}}(\hat{\mathbf{x}})
$$

The scattering state wave function for $k r \ll 1$ outside the DMB scales as

$$
\psi_{\mathbf{k}} \approx \sum_{\ell m} a_{k \ell}(k r)^{\ell} Y_{\ell m}^{*}(\hat{\mathbf{k}}) Y_{\ell m}(\hat{\mathbf{x}}) \approx a_{k 0} Y_{00}^{*}(\hat{\mathbf{k}}) Y_{00}(\hat{\mathbf{x}})
$$

where $a_{k \ell}$ are non-zero numerical coefficients. In other words, for $k R_{(\Phi)} \ll 1$, the $s$-wave term dominates at the boundary of the potential, which can further simplify our calculation. Putting these pieces together, squaring, summing over polarizations and the final state $m$ number and integrating over the photon emission angle, we find the cross section is given by

$$
\sigma_{\gamma, n \ell}=\frac{1}{v} \ell(\ell+1)(2 \ell+1) \frac{Z^{2} \alpha\left|E_{n \ell}\right|}{2 \pi \mu^{2} q^{2}}\left|\int d r r j_{\ell}(q r) R_{n \ell}(r) R_{k 0}^{\prime}(r)\right|^{2} \quad(\ell \geq 1) .
$$

We first note that the above formula could have a broader application beyond the dark matter phenomenology here. The radiative capture cross section in the low energy limit could also be applied to other quantum mechanical systems. Secondly, note that in the simultaneous dipole and low energy limits, both (3.19) and (3.23) reduce to ${ }^{1}$

$$
\sigma_{\gamma, n 1}=\frac{1}{v} \frac{Z^{2} \alpha}{3 \pi}\left|E_{n 1}\right|\left(\left|E_{n 1}\right|+E_{\mathbf{k}}\right)^{2}\left|\int d r r^{3} R_{n 1}(r) R_{k 0}(r)\right|^{2}
$$

\footnotetext{
${ }^{1}$ For eq. (3.23) in the low-energy limit, one can use integration by parts and the Schrödinger equations to derive (3.24).
} 

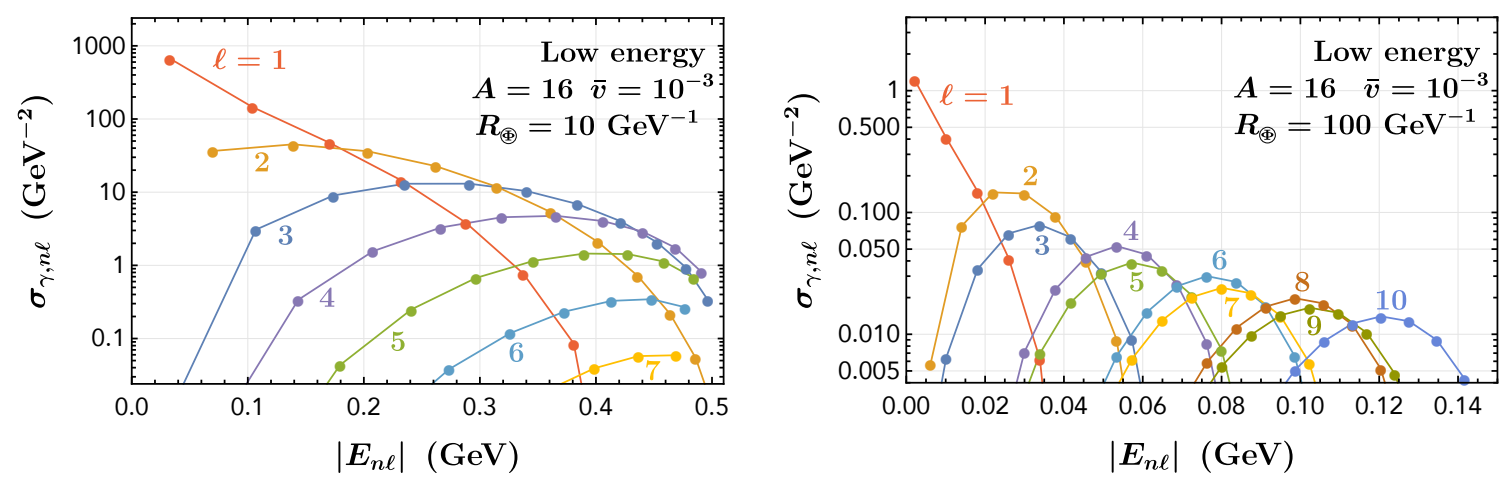

Figure 3. Radiative capture cross section as a function of the binding energy of the bound states for $Z=8, A=16, V_{0}=A \times 32 \mathrm{MeV}$ and $\bar{v}=10^{-3}$, as well as $R_{(\Phi)}=10 \mathrm{GeV}^{-1}$ (left) and $R_{\text {(क) }}=100 \mathrm{GeV}^{-1}$ (right)

In this simultaneous limit, an analytic result for the cross section can be obtained,

$$
\begin{gathered}
\sigma_{\gamma, n 1}=\frac{1}{v} \frac{Z^{2} \alpha}{3 \pi N_{n 1}^{2}} R_{\oplus}^{8}\left|E_{n 1}\right|\left(\left|E_{n 1}\right|+E_{\mathbf{k}}\right)^{2} \times \\
\left\{\frac{c_{3}\left\{\hat{\kappa} \cos \hat{\kappa}\left[\left(\hat{\kappa}^{2}-3 \hat{\kappa}_{n 1}^{2}\right) \sin \hat{\kappa}_{n 1}+\hat{\kappa}_{n 1} \Delta \hat{\kappa}^{2} \cos \hat{\kappa}_{n 1}\right]+\hat{\kappa}_{n 1}^{2} \sin \hat{\kappa}\left(\Delta \hat{\kappa}^{2} \sin \hat{\kappa}_{n 1}+2 \hat{\kappa}_{n 1} \cos \hat{\kappa}_{n 1}\right)\right\}}{\hat{\kappa}\left(\Delta \hat{\kappa}^{2}\right)^{2}\left(\hat{\kappa}_{n 1} \cos \hat{\kappa}_{n 1}-\sin \hat{\kappa}_{n 1}\right)}\right. \\
\left.+\frac{4 \pi\left\{\hat{k}\left[\hat{k}^{2}\left(1+\hat{k}_{n 1}\right)+\hat{k}_{n 1}^{2}\left(3+\hat{k}_{n 1}\right)\right] \cos (\hat{k}-\delta)+\hat{k}_{n 1}^{2}\left(\hat{k}^{2}+\hat{k}_{n 1}^{2}+2 \hat{k}_{n 1}\right) \sin (\hat{k}-\delta)\right\}}{\hat{k}\left(\hat{k}^{2}+\hat{k}_{n 1}^{2}\right)^{2}\left(\hat{k}_{n 1}+1\right)}\right\}^{2}
\end{gathered}
$$

where $\delta$ is the elastic scattering phase, given in our convention by $\tan \delta=c_{2} / c_{1}$ from eq. (3.13), $\hat{x}=x R_{(1)}$, and $\Delta \hat{\kappa}^{2}=\hat{\kappa}_{n 1}^{2}-\hat{\kappa}^{2}$. The factor $c_{3}$ is given in (3.14), while $N_{n 1}^{2}$ given in $(3.8)$.

After numerical integration of eq. (3.23), we show the cross sections for two benchmark radii in figure 3 for illustration, $R_{(\text {() }}=10 \mathrm{GeV}^{-1}$ and $R_{(\text {() }}=100 \mathrm{GeV}^{-1}$, along with the oxygen element and $\bar{v}=10^{-3}$. For $R_{\oplus}=10 \mathrm{GeV}^{-1}$, the energy levels can be found in the left panel of figure 1 , while the $R_{(\Phi)}=100 \mathrm{GeV}^{-1}$ case shows similar behaviors. The most excited $p$-wave state has $E_{11} \approx 32.7 \mathrm{MeV}$ for $R_{\text {(क) }}=10 \mathrm{GeV}^{-1}$ and $E_{11} \approx 2.2 \mathrm{MeV}$ for $R_{(\Phi)}=100 \mathrm{GeV}^{-1}$. As can be seen from figure 3 , the radiative capture cross section is dominated by the most excited state of $\ell=1$ bound states, which is precisely the limit in which the dipole approximation applies. For a fixed $\ell$, the cross section decreases exponentially for deeper bound states with larger binding energy.

In figure 4 , we show the capture cross section as a function of $R_{\oplus}$ up to a radius slightly smaller than $2 \pi / k \approx 2 \pi /\left(A m_{p} \bar{v}\right) \approx 400 \mathrm{GeV}^{-1}$. Again, one can see a clear oscillation behavior, which is due to the resonance effects in the scattering state. The cross section envelope has a mild dependence on the radius, although it is very sensitive to the actual value of $R_{(\Phi)}$ within one period of the wave. 

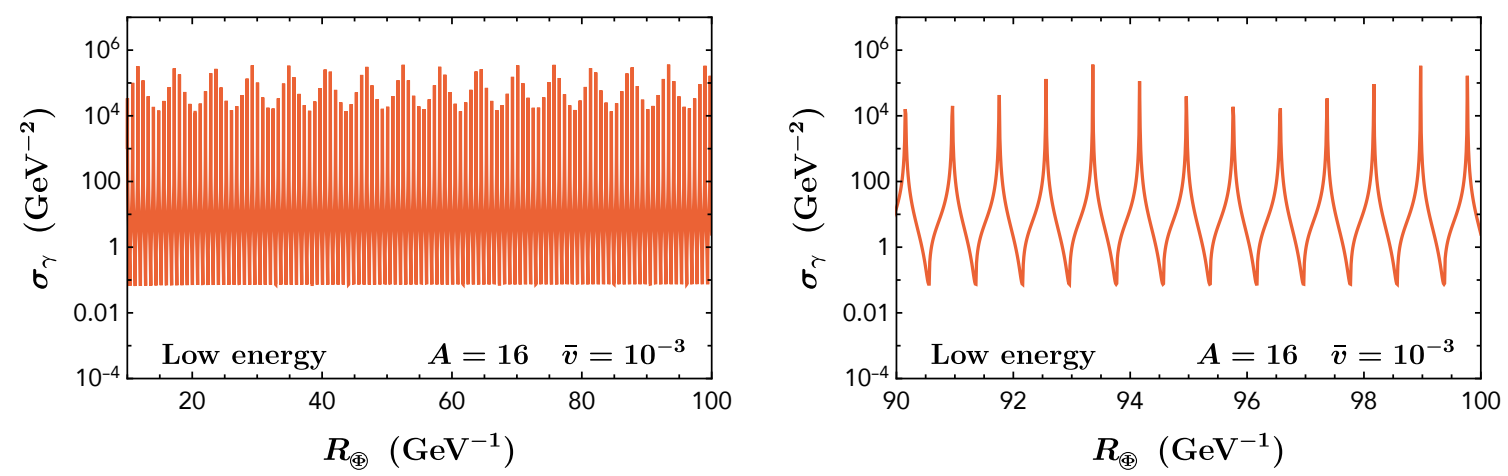

Figure 4. Radiative capture cross section as a function of the DMB radius for $Z=8, A=16$, $V_{0}=A \times 32 \mathrm{MeV}$, and $\bar{v}=10^{-3}$ in the low energy limit, in which only $s$-wave scattering state is included. The right panel narrows the range to the largest radii considered.

It is instructive to compare the radiative capture cross section to the elastic scattering cross section. Using the phase shift method, the elastic scattering cross section is calculated in the low energy limit by

$$
\sigma_{\text {elastic }} \approx \frac{4 \pi}{\kappa^{2}}\left[\tan \left(\kappa R_{(\Phi)}\right)-\kappa R_{\oplus}\right]^{2}
$$

which has a similar oscillating behavior with the same periodicity. The ratio of the radiative capture cross section (in the region under computational control) to this value is shown in figure 5, which still has an oscillating behavior. In the dashed and black lines, we guide the general envelop behavior of this ratio. The general behavior of this ratio as a function of $v$ and $R_{\oplus}$ has a simple scaling

$$
\sigma_{\gamma} / \sigma_{\text {elastic }} \propto v^{-1} R_{\oplus}^{-3 / 2}
$$

with the range of radii satisfying the low energy approximation.

Note that as $R_{\oplus \rightarrow}$ is varied, the scattering wave function in each partial wave mode can be resonantly enhanced when

$$
R_{\text {(1) }} \approx \frac{J_{\ell-1 / 2, n}}{\kappa},
$$

where $J_{\nu, n}$ are Bessel function zeros. The $s$-wave wave function gets enhanced by a factor of $\kappa / k$, leading to an enhancement of the radiative capture cross section by $(\kappa / k)^{2}$.

The dependence on the DMB radius of the total radiative capture cross section in the dipole limit is shown in figure 2. Beyond $R_{\oplus} \approx 12 \mathrm{GeV}^{-1}$, we work in the low energy approximation. The dependence on $R_{(\Phi)}$ in this limit is shown in figure 4 . Beyond $R_{(\Phi)} \approx$ $100 \mathrm{GeV}^{-1}$, the low energy limit is no longer applicable.

\subsection{Large radius limit}

In the large radius limit, the approximations we have used cease to apply and the calculation of the radiative capture cross section becomes computationally prohibitive. The elastic scattering cross section, on the other hand, can be determined in this limit by summing 


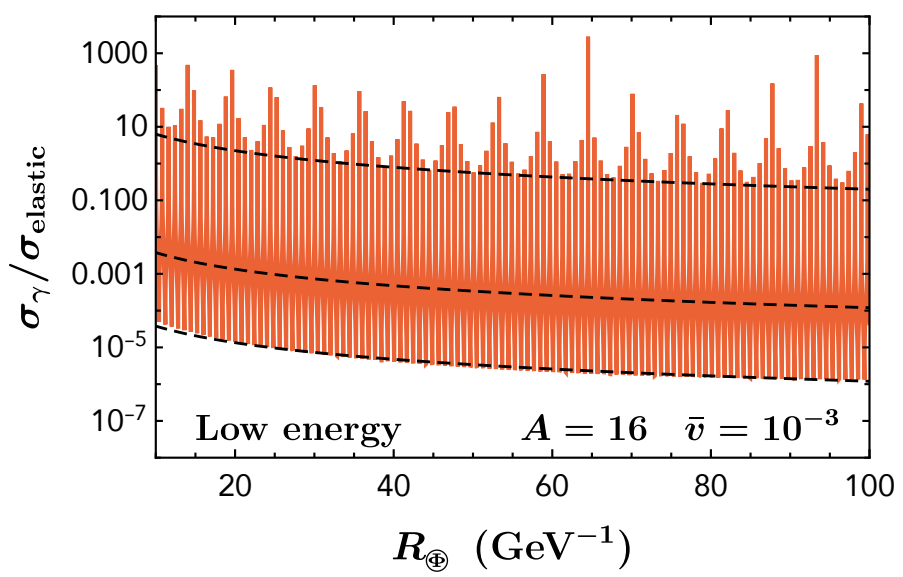

Figure 5. Ratio of the radiative capture to elastic scattering cross section of DMB in the low energy limit. The benchmark $Z=8, A=16, V_{0}=A \times 32 \mathrm{MeV}$ and $\bar{v}=10^{-3}$ is used. The dashed and black line has the ratio $\propto R_{(\Phi)}^{-3 / 2}$.

our analytic expression for the partial wave cross section to a sufficiently high partial wave number. As seen in ref. [11], it saturates the geometric cross section $\pi R_{\oplus}^{2}$ up to an $\mathcal{O}(1)$ factor. We proceed by estimating the ratio of the radiative capture cross section to the known elastic cross section in two different ways: by extrapolating the ratio shown in figure 5 to large radius and by determining this ratio in neutron capture data. Neither procedure is entirely robust, but they are meant to provide a guideline for the possibilities.

In the low energy limit, we have found that the ratio of the radiative capture to elastic scattering cross sections scales as $R_{\oplus}^{-3 / 2}$. Extrapolating this behavior to large $R_{\oplus}$ indicates that the radiative capture cross section scales as $R_{(\Phi)}^{1 / 2}$. We estimate that for $k R_{(\Phi)} \gg 1$ the $\sigma_{\gamma}$ should saturate to

$$
\sigma_{\gamma} \sim 60 \mathrm{GeV}^{-2} \times\left(\frac{10^{-3}}{v}\right)\left(\frac{R_{\text {(†) }}}{10^{5} \mathrm{GeV}^{-1}}\right)^{1 / 2}
$$

Alternatively, this ratio can be estimated from neutron radiative capture data (see figure 6). The data are plotted as a function of the incident neutron momentum. Since the relevant comparison for determining the large $R_{\oplus}$ limit is $k R_{\oplus} \gg 1$, the limit is expected to be reached at large kinetic energy when the momentum becomes comparable to the effective inverse radius of the nucleus. Only for relatively heavy elements does radiative capture reach the large $k R_{(\Phi)}$ limit below the complicated $\mathrm{MeV}$ scale. The data are not sufficiently homogeneous across different nuclei to determine a clear numerical pattern. Nevertheless, the ratios are seen to follow the expected qualitative behavior, going to a smooth function in the large $k R_{(\Phi)}$ limit (the region to the right of the resonance region). The ratios of radiative capture to elastic scattering cross sections for isotopes of uranium, tungsten and silver are shown in figure 6 using the TENDL-2017 model [26, 27]. These nuclei are chosen as cases where there is a significant amount of data that agree with the model, as the quality and availability of data varies widely between different isotopes. 


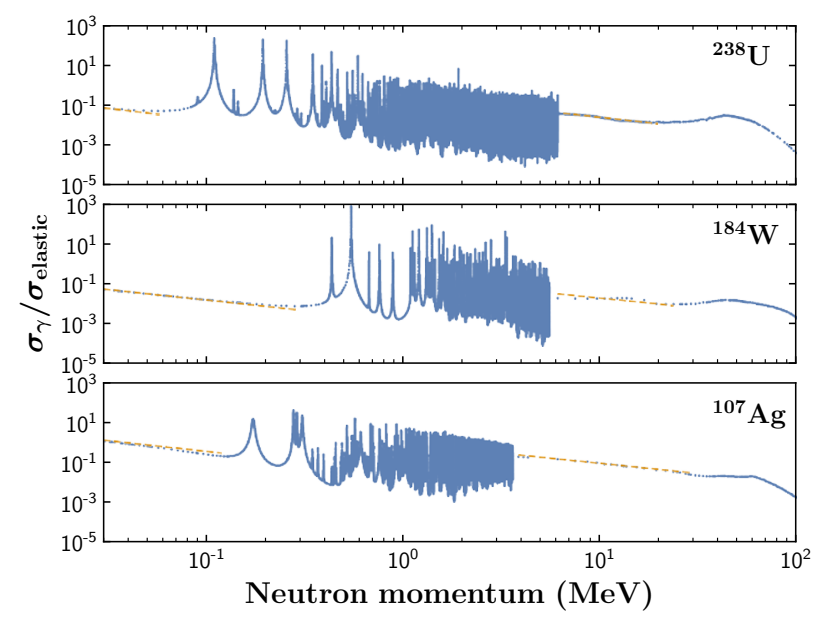

Figure 6. Ratio of the radiative capture to elastic cross sections for neutron capture by three different nuclei using the TENDL-2017 model $[26,27]$ in blue color. The dashed orange lines indicate a $1 / v$ behavior expected at energies below the nuclear resonances and at energies above the nuclear resonances, but below onset of free nucleon scattering.

\section{Prospects for detection}

Radiative capture of nuclei by MDM entering the detector deposits significantly more energy than elastic scattering. The radiation from the initial capture is seen in figure 3 to be of order $\mathrm{MeV}$ or larger. Furthermore, excited states are typically produced; their subsequent decay leads to additional emission totaling around $100 \mathrm{~s} \mathrm{of} \mathrm{MeV}$.

A full study of these signals in individual detectors is beyond the scope of this work. Nevertheless, we consider some basic properties of current and forthcoming detectors to determine the viability of this signal. Direct detection experiments such as Xenon1T [28] and LZ [29] should be sensitive to radiative capture as the deposited energy far exceeds their threshold. Current analyses veto multiple energy deposits in a short time window [30]. A single energy deposit would likely be beyond their current search window, but should be visible if it exceeds the radioactive background. More striking would be several deposits of comparable energies. We consider this multi-hit signal as our primary signal.

The main advantage of considering radiative capture signals, however, is to consider higher energy threshold, but larger detectors. These detectors are primarily large neutrino detectors. The threshold in the full IceCube volume is at the $100 \mathrm{GeV}$ scale [31], above the energies typically deposited by MDM. The next largest that could be sensitive is Hyper-Kamiokande [32]. A few MeV energy deposit, which is the energy released in the initial capture, is close to the threshold and, even if it is reconstructable, may not be distinguishable from radioactive and other backgrounds if it is isolated. We therefore put the requirement that at least 5 capture events occur during MDM passage through the detector for all detectors considered for our main analysis. This could be particularly striking at detectors with tracking capabilities like DUNE [33], where the deposits would 
form a line in the detector. Highly segmented detectors like $\mathrm{NO} \nu \mathrm{A}$ [34] may also be able to track the MDM passage in 2D.

A multi-hit signal would be a spectacular event that would be hard to fake with any background. It may, however, not be required in order to identify an event with radiative capture during MDM passage. The radiative capture event typically produces a highly excited bound state, which de-excites and leads to further photon production. This proceeds until the ground state is reached with a binding energy of $100 \mathrm{~s}$ of $\mathrm{MeV}$, that is with the release of $100 \mathrm{~s}$ of $\mathrm{MeV}$ in photons. Such photons are unlike the dominant potential backgrounds from solar and atmospheric neutrinos in their energy spectrum and topology respectively, while they are above the radioactive decay energy range. Cosmic rays could be an additional background for surface detectors like ProtoDUNE [35], though the cosmic ray tagger should reduce this background significantly. If the search can indeed be made background free by judicious selection criteria, then a very small number of radiative capture single-hit events would be required for a discovery. The expected event rate scales like $1 / M_{\text {(क) }}$ until a point is reached that the number of MDM passing through the detector over the course of the experiment running time is less than one. At that point, corresponding to an upper bound on $M_{\text {(D) }}$, expected sensitivity is lost. A detailed study of the feasibility of such a low event count search is beyond the scope of this work.

We now comment further on the capabilities of water Cherenkov, liquid argon time projection chamber (LArTPC), and liquid scintillator detectors to detect photons of the relevant energies. In any of these detectors, the visible result of the photon that is emitted during radiative capture is an electron/positron produced by Compton scattering at low energies or $e^{+} e^{-}$pair production above $1 \mathrm{MeV}$. The remaining question is what is the threshold for detecting the charged particles produced in these processes.

The largest suitable water Cherenkov detector at the moment is Super-Kamiokande [36]. Another detector, Hyper-Kamikande, with similar technology, but an order of magnitude larger mass and volume is planned. The physical threshold is given by the Cherenkov momentum of the electron in water, namely $p=583 \mathrm{keV}$. In principle, any photon above the corresponding Cherenkov kinetic energy of $263 \mathrm{keV}$ can produce a visible electron. The efficiency for detection at these low energies is likely to be poor, but if multiple such events are lined up through the detector, the detection prospects may be improved. In practice, the lowest threshold analyses have pushed down to electron kinetic energies of $3.5 \mathrm{MeV}$ [37].

LArTPC is an up-and-coming detector technology. The largest such detector will be the Deep Underground Neutrino Experiment (DUNE). Other smaller detectors based on this technology, such as ProtoDUNE [35], are currently operating. In between sits the ICARUS experiment [38], slated to start taking data in December 2019. These detectors track charged particles as they pass through the Argon. The threshold energy at DUNE and in LArTPC detectors in general is less well known as reconstruction in these detectors is still under active development. The limit is set by the travel distance of the electron in liquid Argon. The TPC wire pitch is roughly $4 \mathrm{~mm}$ at DUNE, so the electron should travel at least that distance to have multiple hits, corresponding to a kinetic energy of around $900 \mathrm{keV}$. In order to reconstruct particles, at least 10 hits are generally required. 


\begin{tabular}{|lcccc|}
\hline Detector & $A_{\text {eff }}\left(\mathrm{cm}^{2}\right)$ & $L_{\text {eff }}(\mathrm{cm})$ & Nuclei & $n_{A}\left(10^{22} \mathrm{~cm}^{-3}\right)$ \\
\hline Xenon 1T & $1.09 \times 10^{5}$ & 64.2 & $\mathrm{Xe}$ & 1.42 \\
LZ & $2.65 \times 10^{5}$ & 100 & $\mathrm{Xe}$ & 1.42 \\
ICARUS & $5.08 \times 10^{5}$ & 255 & $\mathrm{Ar}$ & 2.10 \\
ProtoDUNE & $6.78 \times 10^{5}$ & 446 & $\mathrm{Ar}$ & 2.10 \\
Borexino & $5.67 \times 10^{5}$ & 567 & $\mathrm{C}$ & 3.96 \\
No $\nu$ A & $1.17 \times 10^{7}$ & 1400 & $\mathrm{C}$ & 3.10 \\
JUNO & $9.84 \times 10^{6}$ & 2360 & $\mathrm{C}$ & 3.79 \\
Super-Kamiokande & $1.88 \times 10^{7}$ & 2670 & $\mathrm{O}$ & 3.34 \\
DUNE & $2.14 \times 10^{7}$ & 2290 & $\mathrm{Ar}$ & 2.10 \\
Hyper-Kamiokande & $1.12 \times 10^{8}$ & 4580 & $\mathrm{O}$ & 3.34 \\
\hline
\end{tabular}

Table 1. Properties of the detectors considered in our estimation of MDM discovery potential.

In other words, the electron should travel at least $40 \mathrm{~mm}$, corresponding to a kinetic energy of around $9 \mathrm{MeV}$. On the other hand, the DUNE CDR sets a $e^{ \pm} / \gamma$ threshold at $30 \mathrm{MeV}$ [33], as low energy electromagnetic particles may become difficult to disentangle from other charged particles such as muons. Above $30 \mathrm{MeV}$, an electron will typically undergo Bremsstrahlung before stopping, leading to a characteristic shower.

The largest liquid scintillator detectors are currently Borexino [39] and the $\mathrm{NO} \nu \mathrm{A}$ [34] far detector. JUNO [40], a forthcoming detector that also uses a liquid scintillator, will be significantly larger. The medium in the latter is a linear alkyl benzene (LAB) that is planned to have excellent energy resolution. The LAB is $88 \%$ carbon by mass. The low energy reconstruction is limited by the requirement of a minimum number of photoelectrons to reconstruct the radiative capture event. The threshold for the trigger can be as low as $0.065 \mathrm{MeV}$ [41] and easily below $0.5 \mathrm{MeV}$ [42], which should easily be able to detect a radiative capture event. Borexino has a similarly low threshold [39]. The $\mathrm{NO} \nu \mathrm{A}$ detector is designed for higher energy events and a threshold around $15 \mathrm{MeV}$ [34]. This would likely make it challenging to observe the capture photon, but the de-excitation photons could still be seen.

The properties of the detectors, including geometry and the largest nuclei that make up a significant fraction of the medium are also provided in table 1. The effective area $A_{\text {eff }}$ of the detector is the average area normal to the DM trajectory over the DM velocity distribution and uniform position distribution. The effective length $L_{\text {eff }}$ is the average length of the DM path through the detector. For detectors with multiple modules, it is assumed that the modules are sufficiently closely spaced that they operate functionally as a single large detector. Radiative capture is dominated by the most massive common nuclei in the detector, so we consider only interactions with these nuclei. The number density of the dominant nuclei are denoted by $n_{A}$.

Given this analysis strategy, we proceed to determine the region of parameter space to which each of these detectors is sensitive. We parameterize the models in terms of the 


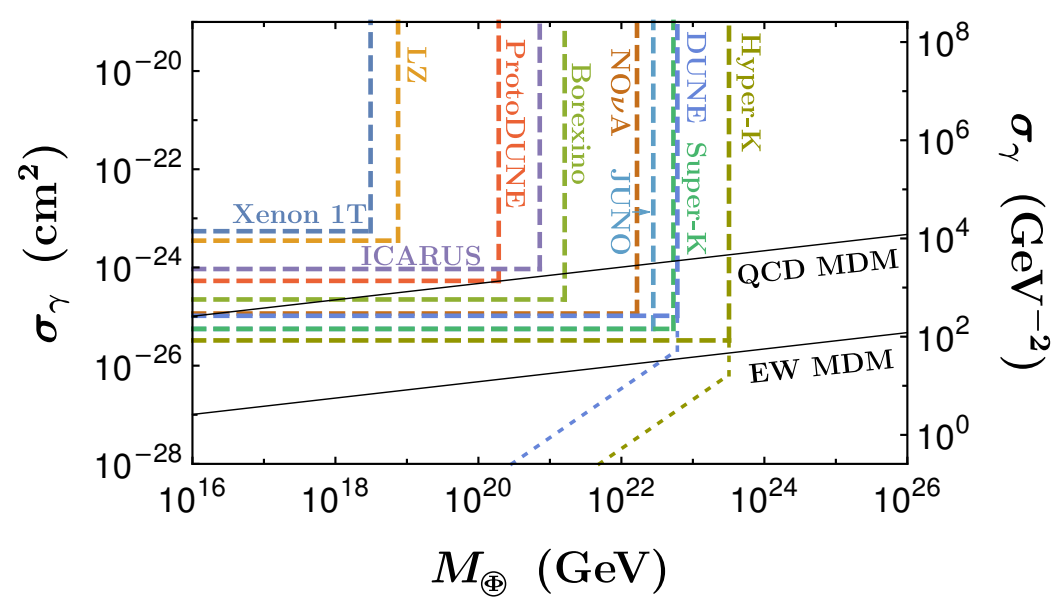

Figure 7. Sensitivity of Xenon 1T (blue) [28], LZ (orange) [29], ProtoDUNE (red) [35], ICARUS (purple) [38], Borexino (green) [39], NO $\nu$ A (brown) [34], JUNO (light blue) [40], Super-Kamiokande (teal) [36], DUNE (dark blue) [43], and Hyper-Kamiokande (dark green) [32] to radiative capture of nuclei by MDM. The experiments are listed in order of increasing mass sensitivity. The dashed lines indicate the sensitivity if at least 5 radiative capture events are required for each MDM passage through the detector. A running time of 10 years is assumed for Borexino, Super-Kamiokande, DUNE, and Hyper-Kamiokande, 5 years is assumed for ICARUS and $\mathrm{NO} \nu \mathrm{A}$ and one year at the direct detection experiments and ProtoDUNE. The dotted lines for DUNE and Hyper-Kamiokande indicate the region in which at least one radiative capture event is expected over the whole running time. The black line indicates the estimated radiative capture cross section for QCD and EW density MDM.

MDM mass and radiative capture cross section of the heaviest nucleus in the detector in question in order to maintain model independence. Pending a detailed study, we assume that 5 energy deposits during the MDM passage is reconstructed with $100 \%$ efficiency and is efficiently separable from potential backgrounds such as radioactive decays, cosmic rays and neutrinos. The resulting estimated sensitivity is presented in figure 7 . We assume a one year running time for Xenon 1T, LZ, and ProtoDUNE, a 5 year running time at ICARUS and $\mathrm{NO} \nu \mathrm{A}$ and a 10 year running time for the remaining large neutrino experiments. In addition, for the DUNE and Hyper-Kamiokande experiments, we indicate the region in which at least one radiative capture event will occur over 10 year in order to assess the potential sensitivity of a single-hit analysis.

\section{Discussion and conclusions}

The signal of radiative capture appears to be rather striking and should be detectable by any of the experiments considered in this work. That said, a full study of the reconstruction of this signal in each experiment is left to future work. In particular, we have left open the question of whether single radiative capture event can be reconstructed and distinguished from background or whether even more distinctive multi-hit events are required in order 
to have a background free search. Furthermore, triggering and data acquisition may be an issue in large, high granularity detectors. While it should be possible to design a system to collect radiative capture events, the current systems may unfortunately miss these events. We also note that searching for radiative capture events is complimentary to performing traditional dark matter direct detection experiments, which are searching for single hit, low energy threshold elastic scattering events. For a large radius, above the threshold to have a bound state, radiative capture events have an advantage because one can utilize a larger detector with a higher threshold. For a small radius, traditional direct detection experiments have an advantage due to their a low energy threshold.

In summary, we have studied the process of radiative capture nuclei by macroscopic dark matter. We have found that the radiative capture process can be comparable to the elastic scattering process, but has generally more promising detection prospects at higher energy threshold neutrino detectors. Current large neutrino detectors such as ProtoDUNE, ICARUS, Borexino, $\mathrm{NO} \nu \mathrm{A}$ and Super-Kamiokande have sensitivity beyond that of direct detection experiments. The next generation of experiments, that is JUNO, DUNE, and Hyper-Kamiokande, should further expand the sensitivity to MDM. A search for multi-hit events would probe parameter space for QCD density MDM such as quark nuggets, while a background free single event analysis could be sensitive to electroweak density MDM such as EWS-DMBs.

\section{Acknowledgments}

We thank Tingjun Yang for useful discussion. The work of Y.B. is supported by the U.S. Department of Energy under the contract no. DE-SC-0017647. The work of J.B. is supported by PITT PACC.

Open Access. This article is distributed under the terms of the Creative Commons Attribution License (CC-BY 4.0), which permits any use, distribution and reproduction in any medium, provided the original author(s) and source are credited.

\section{References}

[1] B. Paczynski, Gravitational microlensing by the galactic halo, Astrophys. J. 304 (1986) 1 [INSPIRE].

[2] K. Griest, Galactic Microlensing as a Method of Detecting Massive Compact Halo Objects, Astrophys. J. 366 (1991) 412 [INSPIRE].

[3] EROS-2 collaboration, Limits on the Macho Content of the Galactic Halo from the EROS-2 Survey of the Magellanic Clouds, Astron. Astrophys. 469 (2007) 387 [astro-ph/0607207] [INSPIRE].

[4] H. Niikura et al., Microlensing constraints on primordial black holes with Subaru/HSC Andromeda observations, Nat. Astron. 3 (2019) 524 [arXiv:1701.02151] [InSPIRE].

[5] E. Witten, Cosmic Separation of Phases, Phys. Rev. D 30 (1984) 272 [InSPIRE].

[6] X. Liang and A. Zhitnitsky, Axion field and the quark nugget's formation at the QCD phase transition, Phys. Rev. D 94 (2016) 083502 [arXiv:1606.00435] [INSPIRE]. 
[7] Y. Bai and A.J. Long, Six Flavor Quark Matter, JHEP 06 (2018) 072 [arXiv:1804.10249] [INSPIRE].

[8] R. Friedberg, T.D. Lee and A. Sirlin, A Class of Scalar-Field Soliton Solutions in Three Space Dimensions, Phys. Rev. D 13 (1976) 2739 [INSPIRE].

[9] S.R. Coleman, Q Balls, Nucl. Phys. B 262 (1985) 263 [Erratum ibid. B 269 (1986) 744] [INSPIRE].

[10] A. Kusenko and M.E. Shaposhnikov, Supersymmetric Q balls as dark matter, Phys. Lett. B 418 (1998) 46 [hep-ph/9709492] [INSPIRE].

[11] E. Pontón, Y. Bai and B. Jain, Electroweak Symmetric Dark Matter Balls, JHEP 09 (2019) 011 [arXiv: 1906.10739] [INSPIRE].

[12] Y. Bai, A.J. Long and S. Lu, Dark Quark Nuggets, Phys. Rev. D 99 (2019) 055047 [arXiv: 1810.04360] [INSPIRE].

[13] M.I. Gresham, H.K. Lou and K.M. Zurek, Nuclear Structure of Bound States of Asymmetric Dark Matter, Phys. Rev. D 96 (2017) 096012 [arXiv:1707.02313] [INSPIRE].

[14] D.M. Grabowska, T. Melia and S. Rajendran, Detecting Dark Blobs, Phys. Rev. D 98 (2018) 115020 [arXiv: 1807.03788] [INSPIRE].

[15] D.M. Jacobs, G.D. Starkman and B.W. Lynn, Macro Dark Matter, Mon. Not. Roy. Astron. Soc. 450 (2015) 3418 [arXiv: 1410.2236] [INSPIRE].

[16] J. Bramante, B. Broerman, R.F. Lang and N. Raj, Saturated Overburden Scattering and the Multiscatter Frontier: Discovering Dark Matter at the Planck Mass and Beyond, Phys. Rev. D 98 (2018) 083516 [arXiv: 1803.08044] [INSPIRE].

[17] J. Bramante, B. Broerman, J. Kumar, R.F. Lang, M. Pospelov and N. Raj, Foraging for dark matter in large volume liquid scintillator neutrino detectors with multiscatter events, Phys. Rev. D 99 (2019) 083010 [arXiv: 1812.09325] [INSPIRE].

[18] H.A. Kramers, XCIII. On the theory of X-ray absorption and of the continuous X-ray spectrum, Lond. Edinb. Dubl. Phil. Mag. J. Sci. 46 (1923) 836.

[19] V.M. Katkov and V.M. Strakhovenko, Radiative recombination and its application in experiments on electron cooling, Sov. JETP 48 (1978) 639.

[20] K. Olaussen, H.A. Olsen, I. Overbo and P. Osland, Proton capture by magnetic monopoles, Phys. Rev. Lett. 52 (1984) 325 [INSPIRE].

[21] B. Allen, I. Bergovist, R. Chrien, D. Gardner and W. Poenitz, Neutron radiative capture, Pergamon Press, Oxford U.K. (1984).

[22] H.-Y. Cheng and C.-W. Chiang, Revisiting Scalar and Pseudoscalar Couplings with Nucleons, JHEP 07 (2012) 009 [arXiv:1202.1292] [INSPIRE].

[23] J.M. Alarcon, L.S. Geng, J. Martin Camalich and J.A. Oller, The strangeness content of the nucleon from effective field theory and phenomenology, Phys. Lett. B 730 (2014) 342 [arXiv: 1209.2870] [INSPIRE].

[24] H. An, M.B. Wise and Y. Zhang, Effects of Bound States on Dark Matter Annihilation, Phys. Rev. D 93 (2016) 115020 [arXiv:1604.01776] [INSPIRE].

[25] J.J. Sakurai, Modern quantum mechanics, Addison-Wesley, Reading MA U.S.A. (1994). 
[26] A.J. Koning and D. Rochman, Modern Nuclear Data Evaluation with the TALYS Code System, Nucl. Data Sheets 113 (2012) 2841 [INSPIRE].

[27] N. Soppera, M. Bossant, O. Cabellos, E. Dupont and C.J. Díez, JANIS: NEA JAva-based Nuclear Data Information System, EPJ Web Conf. 146 (2017) 07006.

[28] XENON collaboration, The XENON1T Dark Matter Experiment, Eur. Phys. J. C 77 (2017) 881 [arXiv : 1708.07051] [INSPIRE].

[29] LZ collaboration, The LUX-ZEPLIN (LZ) Experiment, Nucl. Instrum. Meth. A 953 (2020) 163047 [arXiv: 1910.09124] [INSPIRE].

[30] XENON collaboration, XENON1T Dark Matter Data Analysis: Signal Reconstruction, Calibration and Event Selection, Phys. Rev. D 100 (2019) 052014 [arXiv:1906.04717] [INSPIRE].

[31] ICECuBE collaboration, Probing neutrino emission at GeV energies from compact binary mergers with IceCube, PoS (ICRC2019) 865 (2020) [arXiv: 1908.08299] [INSPIRE].

[32] Hyper-Kamiokande Working Group, A Long Baseline Neutrino Oscillation Experiment Using J-PARC Neutrino Beam and Hyper-Kamiokande, arXiv:1412.4673 [INSPIRE].

[33] DUNE collaboration, Long-Baseline Neutrino Facility (LBNF) and Deep Underground Neutrino Experiment (DUNE), arXiv:1512.06148 [INSPIRE].

[34] NOvA collaboration, The NOvA Technical Design Report, FERMILAB-DESIGN-2007-01 (2007) [https://doi.org/10.2172/935497] [INSPIRE].

[35] DUNE collaboration, The Single-Phase ProtoDUNE Technical Design Report, arXiv: 1706.07081 [INSPIRE].

[36] Super-Kamiokande collaboration, The Super-Kamiokande detector, Nucl. Instrum. Meth. A 501 (2003) 418 [INSPIRE].

[37] H. Sekiya, Low energy neutrinos in Super-Kamiokande, J. Phys. Conf. Ser. 718 (2016) 062052 [INSPIRE].

[38] MicroBoone, LAr1-ND and ICARUS-WA104 collaborations, A Proposal for a Three Detector Short-Baseline Neutrino Oscillation Program in the Fermilab Booster Neutrino Beam, arXiv:1503.01520 [INSPIRE].

[39] Borexino collaboration, The Borexino detector at the Laboratori Nazionali del Gran Sasso, Nucl. Instrum. Meth. A 600 (2009) 568 [arXiv:0806.2400] [INSPIRE].

[40] JUNO collaboration, Neutrino Physics with JUNO, J. Phys. G 43 (2016) 030401 [arXiv: 1507.05613] [INSPIRE].

[41] R. Shah, Studies on the trigger configuration for the juno experiment, Master's Thesis, RWTH Aachen University, Aachen Germany (2018).

[42] X. Fang et al., Capability of detecting low energy events in JUNO Central Detector, 2020 JINST 15 P03020 [arXiv: 1912.01864] [INSPIRE].

[43] DUNE collaboration, Long-Baseline Neutrino Facility (LBNF) and Deep Underground Neutrino Experiment (DUNE), arXiv:1601.05471 [INSPIRE]. 